\title{
Les noms sous-spécifiés dans le débat parlementaire : analyse fréquentielle et catégorisation modale
}

\section{Unspecific Nouns in the Parliamentary Debate: Frequency Analysis and Modal Categorization}

\author{
Silvia Adler \\ Université Bar-Ilan \\ Dominique Legallois \\ Université Sorbonne-Nouvelle Paris 3, Clesthia EA 7345
}

\begin{abstract}
Résumé
L'article propose un examen quantitatif de l'usage des noms sous-spécifiés (Nss) dans un genre discursif particulier: le débat parlementaire à l'Assemblée Nationale. On calcule par statistiques, pour les onze constructions auxquelles les Nss participent, la part que chaque construction consacre à chaque nom (force d'attirance), et la part que chaque nom consacre à chaque construction (force de dépendance). Ce calcul permet d'établir la distribution de cette catégorie nominale dans le discours du débat parlementaire. À la suite de ces résultats, on met en évidence les différentes valeurs modales exprimées par les Nss.

Mots-clés : Noms sous-spécifiés, noms généraux, attraction, dépendance, modalités
\end{abstract}

\begin{abstract}
In this paper we propose to examine the use of unspecific (shell) nouns in the French parliamentary debate. We are interested in the frequency of these nouns within the different constructions that host them. Our quantitative analysis, taking into account a set of 11 syntactic constructions, aims to measure the degree of attraction between words and constructions (Attraction and Reliance). This calculation enables to establish the distribution of this nominal category in the corpus, and to highlight the different modal values expressed by the shell nouns.
\end{abstract}

Keywords: Shell nouns, general nouns, attraction, reliance, modalities

\section{OBJECTIFS}

Cet article propose une analyse quantitative et qualitative en corpus d'une catégorie nominale que nous avons qualifiée de sous-spécifiée (Legallois, 2006, Legallois et Gréa, 2006), parfois appelée métaphoriquement, dans les études anglicistes, shell nouns (Schmid, 2000). Les noms sousspécifiés (désormais Nss) sont considérés comme formant un sous-type de noms généraux (Halliday et Hasan, 1976). Pour assurer l'homogénéité de notre analyse, nous travaillerons sur un 
corpus représentatif d'un seul genre discursif ${ }^{1}$ : le débat parlementaire à l'Assemblée Nationale française. Il s'agit donc de proposer un examen de l'usage des Nss dans une pratique langagière pour laquelle la dimension argumentative est évidente. Nous nous intéressons ainsi à la fréquence de ces noms dans les différentes constructions auxquelles ils participent. En effet, jusqu'à présent, les études consacrées aux Nss ont principalement privilégié les seules constructions spécificationnelles (p.ex. Apotheloz, 2008 ; Legallois, 2008). Aussi, nous avons entrepris dans ce travail une analyse quantitative prenant en compte un ensemble de 11 configurations syntaxiques, certaines étant quasi-exclusivement dédiées aux Nss, d'autres acceptant d'autres types nominaux. La démarche suivie consiste à calculer, par statistiques, la part que chacune des onze constructions consacre à chaque nom (Attirance), ainsi que la part que chaque nom consacre à chaque construction (Dépendance). Ces calculs permettent d'apprécier la distribution de cette catégorie nominale dans le discours du débat parlementaire. À partir de ces résultats, nous proposons un classement des Nss selon les valeurs modales de l'épistémique, de l'appréciatif, de l'axiologique, du boulique et du déontique.

\section{LA CLASSE DES NSS PARMI LES NOMS GÉNERAUX}

\subsection{Les noms généraux : aspects fondamentaux}

Le rapport entre la classe des noms généraux et celle des Nss est d'inclusion : cette dernière forme un sous-ensemble de la première. Il est donc nécessaire de revenir dans un premier temps sur les principales caractéristiques des noms généraux, et d'examiner ensuite en quoi les Nss forment une catégorie hyponymique homogène.

Il est rappelé dans l'introduction à ce numéro que Halliday \& Hasan (1976 : 274) donnent pour illustration des noms généraux des lexèmes comme : people, man, woman, child, [human], creature [non-human animate], thing, object [inanimate concrete count], stuff [inanimate concrete mass], business, affair, matter [inanimate abstract], move [action] place [place] question, idea [fact]. Les noms généraux constituent une catégorie nominale particulière pour les raisons suivantes :

A. Leur fréquence est très élevée dans les textes. La fréquence, en tant que telle, n'est pas définitoire de quoi que ce soit; elle constitue cependant un symptôme précieux : cette fréquence élevée est le signe d'un fonctionnement quasi-grammatical, plus exactement quasi-pronominal. Le fonctionnement des noms généraux est alors rapproché de celui des pronoms ; or, il est évident que les pronoms, comme tous les mots « grammaticaux », sont quantitativement remarquables dans les textes.

B. La signification (justement) générale (Mahlberg, 2005) de ces noms est l'une de leurs caractéristiques. De façon plus technique, les noms généraux sont reconnus pour leur faible intension dont la conséquence est logiquement la grande extension. L'observation n'est pas fausse pour des noms comme lieu, sentiment, moment, idée, chose, qui désignent des catégories fondamentalement superordonnées, même si on peut difficilement, comme le remarque R. Huyghe (2015), les considérer comme des hyperonymes :

1. Ils sont entrés dans le bâtiment ; l'endroit était désert ; ? un bâtiment est un type d'endroit (R. Huyghe, $2015: 20$ ).

Mais en toute rigueur, une analyse sémique en termes de sèmes génériques / sèmes spécifiques montre que des noms comme ville, femme possèdent des traits spécifiques, c'est-à-dire distinctifs d'autres items d'un même paradigme. La dimension générale de la signification de ces mots est donc relative. Un critère définitoire plus sûr est l'exploitation de ces noms comme supports de prédication (Adler, 2017). En dehors de tout discours particulier (par ex., didactique), l'intention informationnelle n'est pas de dire, dans les exemples (2) et (3)

2. Marie est une femme de cinquante ans / magnifique

3. Rome est une ville de 3 millions d'habitants / magnifique

\footnotetext{
${ }^{1}$ Contrairement à Schmid (2000) qui travaille sur un ensemble de genres présents dans le BNC.
} 
que :

2'. *Marie est une femme

3'. $\quad$ *Rome est une ville.

La catégorisation est préétablie et ne fait pas l'objet de la prédication.

C. Le fonctionnement pour lequel on peut affirmer qu'un nom est général est celui de la reprise anaphorique :

4. Je connais Rome depuis 20 ans. Cet(te) endroit/lieu/ville est magnifique

5. Je pense que j'irai à Rome pour les vacances. Cet(te) idée/projet/plan/envie me trotte dans la tête depuis longtemps

6. Je connais ma voisine de bureau depuis 20 ans. Cette femme/nana est incroyablement sympathique. 1

C'est ce fonctionnement anaphorique direct - direct en tant que la relation entre l'expression anaphorique et l'expression antécédente n'a pas besoin d'être assertée - qui rapproche fondamentalement la catégorie des noms généraux de celle des pronoms.

Si on considère l'exemple suivant, dans lequel l'expression anaphorique n'a pas pour tête un nom sous-spécifié

6'. Je connais ma voisine de bureau depuis 20 ans.? Cette femen/ générativiste/médaillée olympique est incroyablement sympathique

la transition entre les deux phrases peut être jugée difficile, car peu cohésive en raison de l' «empan conceptuel» entre ma voisine de bureau et la catégorie femen, etc. : la relation demande une interprétation trop importante, la catégorisation devant être explicitement assertée au préalable pour pouvoir fonctionner ${ }^{2}$. Néanmoins, on sait que la présence d'un modifieur, en congruence avec la valeur de l'article démonstratif, permet d'opérer de façon satisfaisante une partition :

6". Je connais ma voisine de bureau depuis 20 ans. Cette générativiste inoxydable est incroyablement sympathique.

Remarquons encore, en ce qui concerne les noms généraux, qu'avec les noms dont les sèmes sont peu spécifiques, la reprise par un nom déterminé par l'article défini est tout à fait naturelle :

4'. Je connais Rome depuis 20 ans. L'/le/ endroit /lieu est magnifique

5'. Je pense que j'irai à Rome pour les vacances. L'/le idée/projet/plan/envie me trotte dans la tête depuis longtemps.

Si la signification du nom est plus spécifique, cette détermination n'est pas naturelle :

4'. Je connais Rome depuis 20 ans. ? La ville est magnifique.

\subsection{Les Nss}

Les noms sous-spécifiés forment une catégorie à part en raison des points suivants :

A. Ce sont des noms abstraits. Ils se différencient donc des noms comme homme, femme, endroit, etc. Ils sont comptables mais cette propriété semble partagée par tous les noms généraux. Ils sont admis au pluriel, ainsi que dans les exclamatives en que sans que l'on puisse dire qu'ils sont extensifs au sens de Flaux \& Van de Velde (2000) :

7. Que d'idées/ problèmes/objectifs/principes !

B. Les Nss sont pleinement pris en charge par le locuteur, ce dont rend compte la modification modale par vrai ou véritable ou l'emploi exclamatif (Adler, 2012) :

8. c'est un(e) vrai(e) problème, objectif, principe, solution, question, etc. / Quel problème ! etc.

Seuls les adjectifs ou pronoms nominalisés (l'essentiel, l'important, le tout, etc.) ne peuvent être modifiés.

C. Les antécédents des noms sous-spécifiés sont des entités de $2^{\mathrm{e}}$ ou de $3^{\mathrm{e}}$ ordre selon la terminologie de Lyons (1977), c'est-à-dire constituent des situations, événements ou procès, ou

${ }^{2}$ On peut se référer à Schnedecker (2006) pour une étude sur la transition référentielle de ce type. 
encore des faits, des propositions (des objets linguistiques ou textuels : question, argument, etc.). Par conséquent, ces noms ne peuvent jamais se rapporter à des individus.

D. On considère généralement que la sous-spécification de ces noms est d'ordre sémantique : leur signification est générale. Cela est vrai pour les noms les plus fréquents, mais reste à relativiser pour des emplois qui, s'ils sont moins nombreux, ne sont pas marginaux. Dans notre corpus, on relève par exemple le nom scandale dont on ne voit pas en quoi il serait, sémantiquement, peu spécifié. Selon nous, la sous-spécification est avant tout dénotationnelle dans la mesure où il n'est guère possible de déterminer l'image conceptuelle d'un objet qui serait un dénoté prototypique (quelle est l'image d'une idée, d'une solution, d'un intérêt, d'un scandale ?) ; certes, la raison de cela s'explique en partie par la dimension abstraite du nom, mais il convient d'ajouter que ces lexèmes constituent en discours des rôles en recherche de valeurs. Aussi, comme l'a remarqué Blanche-Benveniste (1992), les valeurs sont l'objet des questions formulées en quel est le $N$ ? :

9. Quel(le) est le problème/la question/l'objectif/l'enjeu/la situation, etc. ?

Une telle formulation se montre impossible pour tout autre nom, général ou non, non expansé :

9'. ' *Quel(le) est l'homme/le livre/la ville/le stylo ?

excepté justement si ce nom participe à ce rapport rôle-valeur, en désignant par essence un rôle :

9'. $\quad$ Quel est le coupable/le vainqueur/le chef ?

E. À l'intérieur de la classe des noms généraux, la classe des Nss semble particulièrement extensible, puisqu'elle accueille non seulement des noms, mais aussi des expressions nominales, telles que le moins que l'on puisse dire, le plus étonnant, le plus grave, le plus important, le plus probable, dont certaines sont convertibles en noms simples : l'étonnant, l'important qui héritent de la valeur superlative.

F. Legallois (2006) et Legallois et Lenepveu (2014) ont montré que certains noms sous-spécifiés constituaient des dénominations naturelles pour les phases des séquences d'enchaînement. Les séquences sont des schémas textuels organisateurs : [OBJECTIF - MISE EN EEUVRE - RÉSULTAT ÉVALUATION], [DÉSIR - MISE EN EUUVRE- SATISFACTION - ÉVALUATION], [SITUATION- PROBLÈME - SOLUTION - ÉVALUATION], [QUESTION - RÉPONSE - ÉVALUATION], etc. Ceci confirme la relation très étroite qui existe entre Nss et textualité.

G. Des observations précédentes, découle le fait que les Nss sont accueillis dans une structure spécificationnelle leur assurant, selon l'expression de Winter (1992), une réalisation lexicale ou, pour Apothéloz (2012), une détermination identificatoire : Le $N_{s s}$ est de Inf. / le $N_{s s}$ est que P. Pour synthétiser les propriétés de ces structures et pour rester dans un cadre hallidayen, on peut dire qu'elles remplissent les fonctions :

idéationnelles dans la mesure où a) elles constituent des dispositifs de spécification du contenu nominal ; b) elles mettent en relation une étiquette nominale et un contenu propositionnel, autrement dit, elles permettent de catégoriser un contenu propositionnel ;

- textuelles (et pragmatiques) dans la mesure où elles assurent à la fois la topicalisation du nom, très souvent accentuée même à l'écrit, avec le pronom de reprise, et la focalisation sur le constituant phrastique ou infinitif, grâce au retardement opéré par le dispositif syntaxique. Par ailleurs, elles assurent également des fonctions organisatrices. Par exemple, l'énumération :

10. Cette loi devait éviter plusieurs écueils. Le premier aurait été de copier les systèmes des pays voisins.

- interpersonnelles, fonctions qui se donnent le plus souvent sous un mode argumentatif, notamment grâce à la valeur modale du nom (cf. section 4) :

11. Il est donc évident que je préférerais débattre avec vous d'un projet socialiste sérieux et intelligible. La vérité est qu'il n'existe pas.

Les constructions spécificationnelles sont manifestement des cas particuliers de constructions pseudo-clivées; à ce sujet, on pourrait penser qu'il y a une correspondance directe entre une «vraie» peudo-clivée et une spécificationnelle. Ceci est exact pour beaucoup de cas, comme : 
12. Ce que nous ne pouvons accepter, c'est que l'on bafoue le droit fondamental de chacun de refuser l'accès et l'inscription de données nouvelles au dossier

exemple pour lequel une nominalisation adjectivale est possible :

12'. L'inacceptable pour nous, c'est que l'on bafoue le droit fondamental [...].

Parfois, cependant, la correspondance proposition/nom n'est pas systématique ; ainsi :

13. Il est faux que la Constitution garantisse la péréquation. Ce qui est vrai, c'est qu'il existe de fortes disparités entre les collectivités locales n'a pas pour exact équivalent :

13'. Il est faux que la Constitution garantisse la péréquation. Le vrai/la vérité, c'est qu'il existe de fortes disparités entre les collectivités locales.

Ces quelques éléments permettent à la fois de justifier l'inclusion des Nss dans la catégorie des noms généraux, et de définir une sous-classe autonome (malgré l'hétérogénéité des Nss). Dans la section suivante, nous présentons le corpus et la méthode suivie pour l'analyse quantitative permettant de définir la force d'attraction entre noms et constructions.

\section{CORPUS ET MÉTHODE}

\subsection{Le corpus}

Le corpus choisi pour cette étude est un ensemble de 28000 interventions à l'Assemblée Nationale française. Ce corpus a été mis à la disposition des participants à la campagne d'évaluation DEFT (défi fouille de textes) de 2007 organisée par le laboratoire Limsi ${ }^{3}$. Il s'agit de transcriptions, dans une visée non linguistique, de textes oraux prononcés par des parlementaires ces textes pouvant avoir fait l'objet d'une préparation préalablement à leur énonciation. Ce corpus de 3874302 mots est original en ce qui concerne l'analyse des Nss, puisque nous ne connaissons pas de travaux portant sur l'analyse de ce type de noms dans les textes oraux. Le corpus a été lemmatisé et catégorisé par le logiciel Cordial. Nous avons utilisé le concordancier CooLox ${ }^{4}$ pour effectuer les requêtes nécessaires et extraire les concordances, puis le script $\mathrm{R}$ "Coll.analysis 3.2a » écrit par S. Gries ${ }^{5}$ pour les calculs statistiques.

\subsection{Méthode}

Dans un premier temps, nous extrayons la fréquence des 100 premiers noms (toutes catégories confondues) du corpus, afin d'apprécier la présence des noms généraux et des Nss. Dans un deuxième temps, nous nous concentrons uniquement sur les Nss. Notre méthode se décline alors en plusieurs étapes :

A. Nous adoptons la démarche employée par Roze, Charnois, Legallois, Ferrari et Salles (2014) qui consiste à extraire et quantifier les Nss du corpus employés dans les constructions spécificationnelles $\boldsymbol{N}_{\text {ss }}$ être de Inf. et $\boldsymbol{N}$ être que $\boldsymbol{P}$. Cela entraine une conséquence méthodologique importante: seuls les Nss apparaissant dans les deux constructions spécificationnelles seront considérés dans les autres constructions, alors que ces dernières peuvent accueillir logiquement d'autres Nss.

B. Nous projetons donc ce lexique en corpus pour identifier les autres constructions dans lesquelles ces noms sont employés. Ces constructions sont aux nombres de 11 : outre les deux constructions spécificationnelles déjà mentionnées, nous considérons :

- la construction spécificationnelle nominale : $N_{s s}\left(c^{\prime}\right)$ est un/le $N$

14. J'ai entendu M. M. X tenir un discours de rassemblement et de dialogue. Le problème, c'est le décalage entre vos intentions et la réalité.

\footnotetext{
${ }^{3}$ Le thème de l'édition était la classification automatique de textes d'opinion. Les actes ont été publiés sur le site https://deft.limsi.fr/actes/2007/. Un des auteurs de cet article a donc participé à cette campagne (Vernier, Mathet, Rioult, Charnois, Ferrari, Legallois, 2007).

${ }^{4}$ https://lipn.univ-paris13.fr/ audibert/lox.php

${ }^{5} \mathrm{http}: / /$ www.linguistics.ucsb.edu/faculty/stgries/
} 
- le groupe nominal $\boldsymbol{N}_{s s} d e$ expansé par un infinitif

15. ce gouvernement aura-t-il le courage de faire un pas supplémentaire ?

- le groupe nominal $\boldsymbol{N}_{s s} q u e$ expansé par une conjonctive

16. Par l'amendement 213 corrigé, nous manifestons notre conviction que le mécanisme de la TIPP flottante est pérenne

- le groupe nominal anaphorique à l'initiale d'une phrase et déterminé par un démonstratif : . $C e \boldsymbol{N}_{s s}$

17. Vous sacrifiez la santé des Français les plus pauvres aux équilibres comptables. Ce choix n'est pas le nôtre.

- $\quad$ la construction exprimant une évaluation : $\boldsymbol{N}_{s s} \boldsymbol{e s t}$ (Modif) Adj.

18. L'argumentation du ministre est techniquement juste. La situation des DOM et celle de la Corse sont profondément différentes.

- la construction introduisant la spécification indépendamment de la syntaxe, à l'aide de $\ll: »: N_{s s}$

19. Le travail est une valeur morale. Il peut donc être sous-payé. La preuve : le pouvoir d'achat des fonctionnaires a été réduit de $3 \%$ !

Cette construction est également employée pour les cas d'énumération (le premier principe : ...). La présence des «: », massive dans le corpus, est un choix du transcripteur puisque le corpus est oral.

la forme exprimant la spécification du nom sous-spécifié : $\boldsymbol{N}_{\text {ss }}$ réside/consiste en (à)

20. plusieurs journaux économiques ont de même souligné le paradoxe qui consiste à ouvrir notre marché à la concurrence.

- la construction impliquant un verbe support. Nous la notons avoir pour $\boldsymbol{N}_{s s}$, mais elle correspond à plusieurs réalisations : avoir pour mission, se fixer comme objectif, avoir l'ambition, etc'.

21. Mon amendement avait pour objectif de combler cette lacune

- $\quad$ la construction être un/le $\boldsymbol{N}_{s s}$ dans laquelle le Nss catégorise ou évalue le sujet

22. On préfère deux entreprises moyennes à une grande entreprise nationale. C'est une erreur stratégique.

Certaines constructions auxquelles participent les noms sous-spécifiés dans le corpus n'ont pas été prises en compte en raison de leur fréquence faible ; par exemple, l'interrogative quel est le Nss? ou encore Tel est le Nss, $\boldsymbol{N}$ être + Interrogatif (le problème est comment répondre à cette question/ quelle stratégie adopter), Nss vouloir que (la logique voudrait que l'on évite de compliquer les procédures).

C. Pour chacune de ces constructions, nous procédons à un double calcul statistique d'association mesurant, premièrement, la part que chacune des onze constructions consacre à chaque nom (Attirance), et, deuxièmement, la part que chaque nom consacre à chaque construction (Dépendance). Le test du Delta P est utilisé (Gries 2013). Si, pour un Nss X et une construction C : $\mathrm{a}=$ la fréquence de la cooccurrence entre $\mathrm{X}$ et $\mathrm{C}$

$\mathrm{b}=$ la fréquence de $\mathrm{X}$ dans le corpus moins la fréquence de $\mathrm{X}$ dans $\mathrm{C}$

$\mathrm{c}=$ la somme de la fréquence de tous les Nss (sauf celle de $\mathrm{X}$ ) apparaissant en cooccurrence avec $\mathrm{C}$ $\mathrm{d}=$ la fréquence de toutes les unités (sauf celle de X) qui apparaissent avec toutes les constructions sauf $\mathrm{C}$. En pratique, on utilise la fréquence totale des mots du corpus.

l'Attraction se calcule : $a /(a+c)-b /(b+d)$

la Dépendance se calcule : $a /(a+b)-c /(c+d)$

${ }^{6}$ Pour une analyse précise de ces expressions, cf. Nakamura (2017). 
Ainsi, théoriquement, un Nss peut être fréquent dans une construction (par rapport à ses autres emplois), mais cette même construction peut « recruter» un grand nombre de Nss, si bien que la Dépendance du Nss à la construction peut être forte, mais l'attraction du Nss par la construction peut être faible.

\section{RÉSULTATS}

\subsection{Les Nss du corpus}

Rappelons que seuls les Nss des 9 autres constructions apparaissant dans les deux constructions spécificationnelles sont retenus dans cette étude. Soit, en tout, 91 noms. Ci-dessous les 10 premiers pour Nss être de :

objectif 70 ; but 36 ; rôle 26 ; priorité 23 ; objet 22 ; problème 22 ; devoir 19 ; essentiel 19 ; moyen 18 ; souci 16.

Et pour Nss est que $P$ :

vérité 45 ; problème 43 ; réalité 23 ; essentiel 14 ; fait 12 ; preuve 10 ; chose 10 ; argument 9 ; différence 8 ; objectif 8 .

Les fréquences ne sont pas spectaculaires. On remarque que le nom projet, figurant au $6^{\mathrm{e}}$ rang des noms les plus fréquents du corpus, n'apparait pas dans le top 10 des deux constructions (non plus d'ailleurs que effet $-11^{\mathrm{e}}$ rang). Ce qui doit être noté, c'est la présence, parmi les 91 noms considérés, de lexèmes que l'on pourrait qualifier de noms sous-spécifiés propres (au genre discursif), par rapport aux noms sous-spécifiés communs. La première catégorie est nommée ainsi pour désigner les noms appartenant aux champs lexicaux développés par le corpus et le genre discursif ; par exemple : projet, mesure, proposition, politique sont des items directement concernés par la nature du corpus. Ainsi, ces noms ont la capacité de participer aux thématiques du débat parlementaire, tout en assurant des fonctions plus générales, comme modaliser et catégoriser un contenu propositionnel :

23. Parallèlement, la politique des pays est de créer des espaces de projet rassemblant élus locaux et sociétés civiles.

Par ailleurs, lorsque projet est complémenté par de loi, il forme un terme technique. Dans ce cas, il perd son fonctionnement de nom sous-spécifié : aucune occurrence de le projet de loi est de Inf. n'est par exemple présente dans le corpus. L'expression a alors besoin d'un nom sous-spécifié (comme vocation ci-dessous), partageant avec elle un sens prospectif :

24. La vocation principale de ce projet de loi est de clarifier les compétences respectives des différents niveaux de collectivités territoriales.

Dans un texte scientifique, méthode, résultat seraient ainsi des Nss propres. Les Nss appelés communs sont, quant à eux, transposables à tout genre discursif (problème, question, vérité, essentiel, etc.).

\subsection{Attraction, dépendance}

Nous donnons ci-dessous les tableaux portant sur la force d'attraction et de dépendance entre Nss et constructions; seuls les scores supérieurs ou égaux à 0.003 (pour l'attraction et pour la dépendance) ont été retenus.

\begin{tabular}{|l|l|}
\hline$N_{s s}$ être de Inf. & frq $=521$ \\
\hline Attraction & $\begin{array}{l}\text { objectif }(0.13) ; \text { but }(0.07) ; \text { rôle }(0.05) ; \text { devoir }(0.04) ; \text { priorité }(0.04) \text {; essentiel } \\
(0.04) ; \text { objet }(0.04) ; \text { problème }(0.04) ; \text { souci }(0.03) ; \text { solution }(0.03) ; \text { moyen }(0.03)\end{array}$ \\
\hline Dépendance & $\begin{array}{l}\text { important }(0.26) ; \text { écueil }(0.15) ; \text { but }(0.1) ; \text { obsession }(0.07) ; \text { devoir }(0.06) ; \\
\text { essentiel }(0.05) ; \text { désir }(0.05) ; \text { raison d'être }(0.05) \text {; enjeu }(0.04) ; \text { mieux }(0.04) ; \\
\text { objectif }(0.03) ; \text { souci }(0.03) ; \text { sagesse }(0.03) ; \text { alternative }(0.03)\end{array}$ \\
\hline
\end{tabular}

\begin{tabular}{|l|l|}
\hline \multicolumn{2}{|l|}{$N_{s s}$ être que $P$. frq $=273$} \\
\hline Attraction & $\begin{array}{l}\text { vérité }(0.16) ; \text { problème }(0.16) \text {; réalité }(0.08) \text {; essentiel }(0.05) ; \text { preuve }(0.04) ; \\
\text { chose }(0.04) ; \text { fait }(0.04) ; \text { important }(0.03) ; \text { différence }(0.03) ; \text { argument }(0.03) ;\end{array}$ \\
\hline
\end{tabular}




\begin{tabular}{|l|l|}
\hline & objectif $(0.03)$; raison $(0.03)$ \\
\hline Dépendance & $\begin{array}{l}\text { important }(0.2) ; \text { vérité }(0.14) \text {; nouveauté }(0.08) ; \text { comble }(0.07) \text {; malheur }(0.06) ; \\
\text { scandale }(0.06) ; \text { inconvénient }(0.05) ; \text { paradoxe }(0.05) \text {; essentiel }(0.04) ; \text { certitude } \\
(0.03) ; \text { pire }(0.03)\end{array}$ \\
\hline
\end{tabular}

\begin{tabular}{|c|c|}
\hline$N_{s s} \hat{e}$ tre un $/$ & Frq $=129$ \\
\hline Attraction & $\begin{array}{l}\text { fait }(0.13) ; \text { problème }(0.13) \text {; objectif }(0.07) \text {; conséquence }(0.06) ; \text { enjeu }(0.05) \\
\text { résultat }(0.05) \text {; raison }(0.05) ; \text { solution }(0.04) ; \text { important }(0.03) ; \text { philosophie } \\
(0.03) ; \text { essentiel }(0.03) \text {; réponse }(0.03)\end{array}$ \\
\hline Dépendance & important $(0.09)$; philosophie $(0.03)$; politique $(0.03)$ \\
\hline
\end{tabular}

\begin{tabular}{|c|c|}
\hline \multicolumn{2}{|c|}{$N_{s s} d e \quad$ frq $=2320$} \\
\hline Attraction & $\begin{array}{l}\text { moyen }(0.19) \text {; volonté }(0.17) \text {; risque }(0.07) \text {; fait }(0.05) \text {; mérite }(0.04) \text {; intention } \\
(0.04) \text {; courage }(0.04) \text {; souci }(0.04) \text {; liberté }(0.03) \text {; décision }(0.03) \text {; façon } \\
(0.03) \text {; raison }(0.03)\end{array}$ \\
\hline Dépendance & $\begin{array}{l}\text { mérite }(0.34) \text {; volonté }(0.3) \text {; courage }(0.27) \text {; désir }(0.19) \text {; souci }(0.18) \text {; honneur } \\
(0.17) \text {; intention }(0.15) \text {; devoir }(0.15) \text {; moyen }(0.14) \text {; souhait }(0.11) \text {; risque } \\
(0.1) \text {; sentiment }(0.1) \text {; certitude }(0.09) \text {; liberté }(0.07) \text {; ambition }(0.07) \text {; but } \\
(0.07) \text {; façon }(0.06) \text {; idée }(0.06) \text {; fait }(0.05) ; \text { manière }(0.05)^{7} \text {; sagesse }(0.05) ; \\
\text { décision }(0.04) \text {; avantage }(0.04) \text {; raison }(0.03) ; \text { choix }(0.03) ; \text { responsabilité } \\
(0.03) \text {; erreur }(0.03)\end{array}$ \\
\hline
\end{tabular}

\begin{tabular}{|l|l|}
\hline$N_{s s}$ que frq=485 \\
\hline Attraction & $\begin{array}{l}\text { fait }(0.6) ; \text { idée }(0.24) ; \text { sentiment }(0.2) ; \text { motif }(0.18) ; \text { preuve }(0.07) ; \text { conviction } \\
(0.06) ; \text { risque }(0.05) ; \text { souhait }(0.04) ; \text { façon }(0.04) ; \text { principe }(0.03)\end{array}$ \\
\hline Dépendance & $\begin{array}{l}\text { sentiment }(0.24) \text {; certitude }(0.21) ; \text { motif }(0.19) ; \text { idée }(0.13) ; \text { souhait }(0.13) ; \text { fait } \\
(0.12) ; \text { conviction }(0.12) ; \text { preuve }(0.06) ; \text { crainte }(0.04)\end{array}$ \\
\hline
\end{tabular}

\begin{tabular}{|l|l|}
\hline. Ce $N_{s s}$ frq $=807$ \\
\hline Attraction & $\begin{array}{l}\text { projet }(0.36) ; \text { mesure }(0.11) ; \text { politique }(0.07) ; \text { choix }(0.05) ; \text { proposition }(0.05) ; \\
\text { question }(0.04) ; \text { décision }(0.04) ; \text { principe }(0.04) ; \text { objectif }(0.02)\end{array}$ \\
\hline Dépendance & projet $(0.05) ;$ obsession $(0.04) ;$ choix $(0.03) ;$ méthode $(0.03)$ \\
\hline
\end{tabular}

\begin{tabular}{|l|l|}
\hline$N_{s s}$ est $A d j . \quad$ & frq=511 \\
\hline Attraction & $\begin{array}{l}\text { projet }(0.2) ; \text { question }(0.1) ; \text { politique }(0.08) ; \text { enjeu }(0.05) ; \text { proposition }(0.05) ; \\
\text { réponse }(0.04) ; \text { conséquence }(0.04) ; \text { solution }(0.04) ; \text { choix }(0.04) ; \text { argument }\end{array}$ \\
\hline Dépendance $(0.03)$ & enjeu $(0.09) ;$ paradoxe $(0.05) ;$ pire $(0.03)$ \\
\hline
\end{tabular}

$N_{s s}: \quad$ frq $=628$

\footnotetext{
${ }^{7}$ Comme le remarque E. Moline, manière (mais sans doute aussi façon et moyen) mériterait un examen à part en raison de son comportement particulier : en effet, si procéder aux élections peut être un problème, cela ne peut en aucun cas être une manière.
} 


\begin{tabular}{|l|l|}
\hline Attraction & $\begin{array}{l}\text { question }(0.14) ; \text { priorité }(0.06) ; \text { chose }(0.06) ; \text { problème }(0.06) ; \text { objectif }(0.05) ; \\
\text { projet }(0.05) ; \text { conséquence }(0.03) ; \text { principe }(0.03) ; \text { argument }(0.03)\end{array}$ \\
\hline Dépendance & $\begin{array}{l}\text { écueil }(0.15) ; \text { obsession }(0.14) ; \text { paradoxe }(0.12) ; \text { certitude }(0.07) \text {; priorité }(0.04) ; \\
\text { chose }(0.04) ; \text { question }(0.03) ; \text { ambition }(0.03) ; \text { enjeu }(0.03) ; \text { but }(0.03) ; \text { crainte } \\
(0.03)\end{array}$ \\
\hline
\end{tabular}

\begin{tabular}{|l|l|}
\hline$N_{s s}$ réside/consiste en $\quad$ frq $=121$ \\
\hline Attraction & $\begin{array}{l}\text { politique }(0.2) ; \text { solution }(0.15) ; \text { méthode }(0.07) ; \text { réponse }(0.07) ; \text { mesure }(0.05) ; \\
\text { projet }(0.04) ; \text { objectif }(0.03)\end{array}$ \\
\hline Dépendance & $\varnothing$ \\
\hline
\end{tabular}

\begin{tabular}{|c|c|}
\hline \multicolumn{2}{|c|}{ avoir pour $N_{s s}$ frq $=2638$} \\
\hline Attraction & $\begin{array}{l}\text { moyen }(0.1) \text {; effet }(0.08) \text {; objectif }(0.06) \text {; sentiment }(0.05) \text {; objet }(0.05) \text {; raison } \\
(0.05) \text {; mérite }(0.04) \text {; courage }(0.04) \text {; conséquence }(0.04) \text {; but }(0.03) \text {; vocation } \\
(0.03) \text {; priorité }(0.03)\end{array}$ \\
\hline Dépendance & 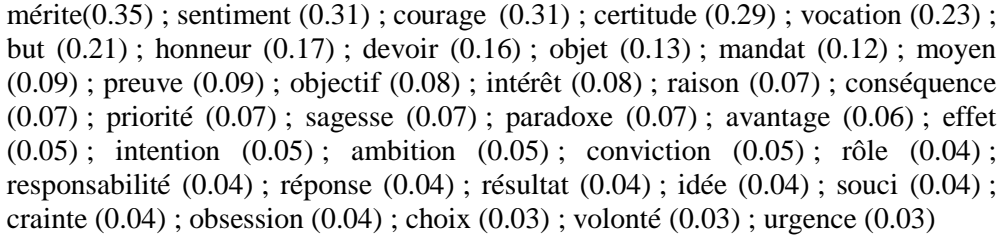 \\
\hline
\end{tabular}

\begin{tabular}{|l|l|}
\hline Est un $N_{s s}$ & frq=995 \\
\hline Attraction & $\begin{array}{l}\text { priorité }(0.06) ; \text { question }(0.06) ; \text { objet }(0.05) ; \text { condition }(0.05) ; \text { conséquence } \\
(0.04) ; \text { raison }(0.04) ; \text { principe }(0.04) ; \text { mesure }(0.04) ; \text { erreur }(0.03) \text {; résultat } \\
(0.03) ; \text { enjeu }(0.03) ; \text { preuve }(0.03) ; \text { objectif }(0.03) ; \text { choix }(0.03) ; \text { problème } \\
(0.03)\end{array}$ \\
\hline Dépendance & $\begin{array}{l}\text { Comble }(0.24) ; \text { scandale }(0.1) ; \text { erreur }(0.08) ; \text { enjeu }(0.08) ; \text { nouveauté }(0.07) ; \\
\text { paradoxe }(0.07) ; \text { priorité }(0.05) ; \text { objet }(0.05) ; \text { preuve }(0.05) ; \text { progrès }(0.04) ; \\
\text { évidence }(0.04) ; \text { devoir }(0.04) ; \text { conséquence }(0.03) ; \text { résultat }(0.03) ; \\
\text { préoccupation }(0.03) ; \text { acte }(0.03) ; \text { vertu }(0.03) ; \text { philosophie }(0.03)\end{array}$ \\
\hline
\end{tabular}

\subsection{Discussion}

Il existe pour l'anglais plusieurs classifications des Nss, notamment celle de Schmid (2000) ou de Flowerdew et Forest (2015). Considérons cette dernière ; elle s'articule autour de 6 catégories :

1- L'expression d'un acte (changement dans le monde) : process, reaction, response, etc. 2l'expression d'une circonstance (l'énoncé répond aux questions : why, how, when, where ?) : way, method, approach, etc. 3- l'expression d'un fait (problem, evidence, fact, etc.). 4- l'expression d'un contenu cognitif (idea, theory, view, etc.). 5- l'expression d'un objet linguistique (question, claim, solution, etc.). 6- l'expression d'une modalité factuelle (responsability, failure, power, etc.) ${ }^{8}$.

Ainsi, en ce qui concerne la construction Nss être de Inf, par exemple, si l'on suit ce classement, les résultats indiquent que cette construction attire des noms appartenant à des catégories diverses, entre autres : l'expression d'un contenu cognitif (objectif, but), l'expression des circonstances (rôle), l'expression d'une modalité factuelle (priorité). Le classement est avant tout

\footnotetext{
${ }^{8}$ Voir leur tableau à la page 116.
} 
référentiel. Autrement dit, ce type de classification ne permettra pas de désigner les dénominateurs communs ou le profil caractéristique et qualitatif des noms intégrant les constructions identifiées, vu la diversité des classes participant à chaque construction. Si l'on convient que les Nss sont pris en charge par l'énonciateur, ce type de classification s'avère d'autant plus insuffisant pour rendre compte de leur distribution. À la recherche d'une classification plus opérante, nous proposons de considérer les Nss comme des expressions fondamentalement modales et de suivre L. Gosselin (2010) et sa définition des différentes modalités. Ainsi, nous considérons que les 6 classes modales (ci-dessous) peuvent rendre compte du fonctionnement des Nss.

- L'aléthique, exprimant la vérité présentée dans ce qu'elle a d'objectif : fait, évidence, chose, logique, vérité, réalité, preuve, résultat, effet, conséquence, condition, façon, méthode, moyen, raison, motif.

Les noms que Flowerdew et Forest associent à l'expression de la circonstance (façon, manière, moyen), sont en fait des unités désignant les conditions de possibilité d'un procès. Aussi, malgré une direction d'ajustement différente, nous les classons ici.

- L'épistémique, exprimant la vérité d'un sujet : sentiment, certitude, crainte, décision, conception, conviction, politique, question, réponse, argument, conclusion, propos, enseignement, mesure.

Question (expression d'un objet linguistique) et réponse (expression d'un acte) participent de l'épistémique car ces noms ont trait au savoir.

- L'appréciatif, exprimant un jugement subjectif, selon ce qui est désirable : comble, essentiel, exigence, important, inconvénient, intérêt, mieux, pire, progrès, urgence, erreur, scandale, drame, paradoxe, écueil, problème, risque, solution, différence, nouveauté.

- L'axiologique, exprimant un jugement de valeur de nature morale, idéologique ou légale : courage, honneur, mérite, sagesse, vertu, habileté, faute.

- Le boulique, exprimant le désir, la volonté, le souhait (la direction d'ajustement est du monde à l'énoncé) : alternative, ambition, but, choix, intention, objectif, objet, préoccupation, désir, volonté, priorité, projet, esprit, idée, proposition.

Un nom comme objectif articule un vouloir et un faire.

- Le déontique, exprimant l'obligatoire, l'interdit, le permis ou le facultatif, selon les institutions : devoir, responsabilité, mission, rôle, principe.

Le croisement des classements s'avère utile pour montrer qu'outre les catégories sémantiques auxquelles appartiennent les noms, il faut considérer aussi leurs valeurs modales intrinsèques. Ainsi, avant d'exprimer un fait, problème marque une appréciation sur une situation.

Pour revenir aux résultats relatifs à la construction Nss être de Inf, cette classification nous permettra d'établir que la construction attire en priorité les noms bouliques (objectif, but, objet), en raison de la direction d'ajustement qu'elle exprime (du monde aux mots). Ce sont en revanche deux termes appréciatifs qui consacrent la plus grande part de leurs emplois à la construction (important, écueil). En ce qui concerne $N_{s s}$ être que $P$, en raison de la direction d'ajustement de la construction (des mots au monde), ce sont logiquement les noms aléthiques (vérité, réalité, fait, chose, preuve) qui sont les plus attirés. Mis à part vérité, ce sont les appréciatifs qui sont les plus engagés dans la construction (Dépendance). Pour ce qui est de $N_{s s}$ être un / le $N$, on remarque par exemple que seul l'appréciatif important consacre une part non négligeable de ses emplois à la construction. Dans la construction $N_{s s}$ de, volonté a une dépendance et une attraction forte. Il est classé parmi les bouliques, mais il n'est pas sans rapport non plus avec les axiologiques (avoir de la volonté, faire preuve de volonté). Du reste, ce sont justement les axiologiques les plus concernés par l'attraction. En ce qui concerne $N_{s s}$ que, le très fort taux d'attraction de fait s'explique par une grammaticalisation de l'expression le fait que (le fait de/que sont des introducteurs qui servent à la 
nominalisation de l'infinitif ou de la proposition) ${ }^{9}$. Les épistémiques se montrent plus particulièrement attirés par la construction [. $\left.C e N_{s s}\right]$. Par ailleurs projet développe une affinité évidente pour la construction, tant pour l'attraction que pour l'attirance. Si on projette les noms sur un graphe, en abscisse l'attraction, en ordonnée la dépendance, on observe donc que projet est localisé seul en haut et à droite - la place de choix pour corréler attraction et dépendance :

- Fig. 1 : Corrélation entre l'Attraction et la Dépendance pour la construction [. $\mathrm{Ce}$ $\left.N_{s s}\right]$

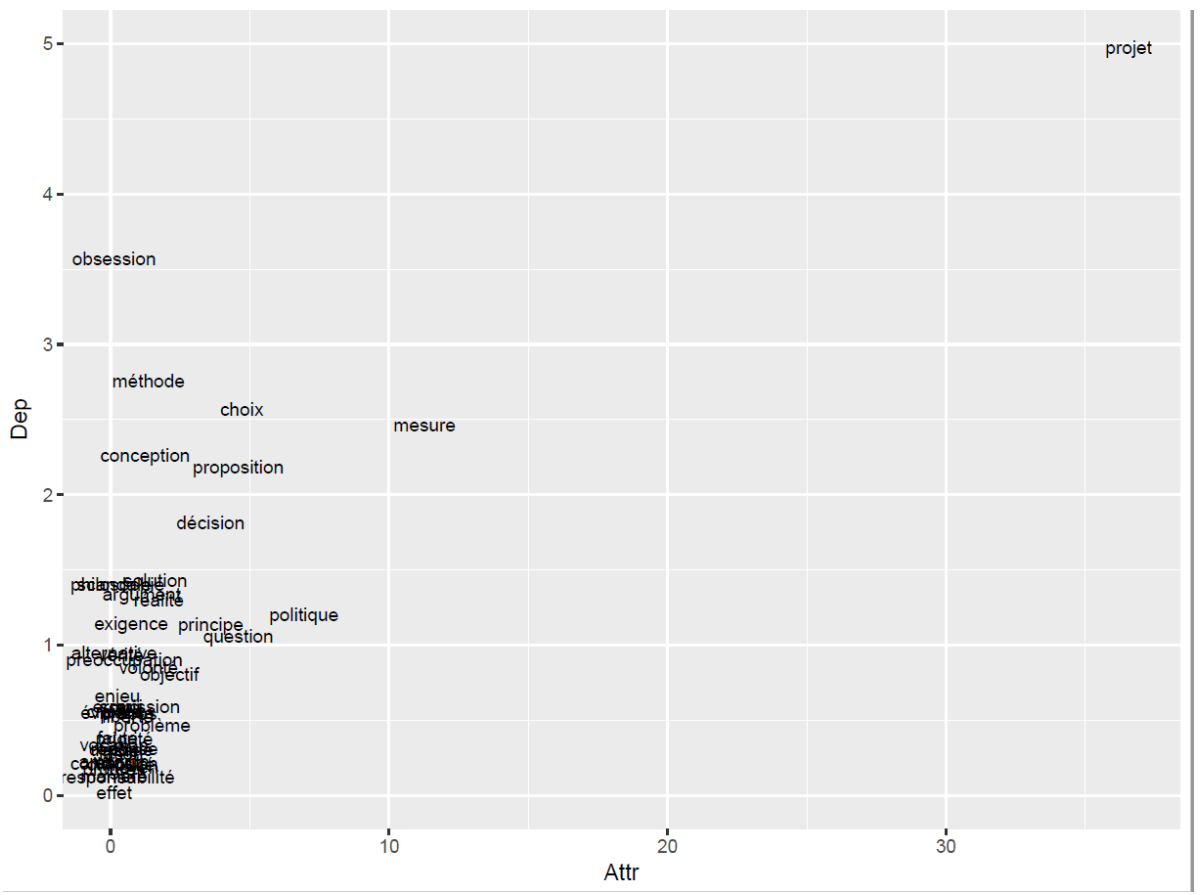

Projet est aussi fortement attiré par la construction $N_{s s}$ est Adj. Le débat parlementaire consiste bien sûr à évaluer les projets discutés. On remarque donc ici une prédilection pour les noms sousspécifiés propres, qui ont un rapport étroit à la pratique et à la terminologie professionnelles.

Dans la construction $\mathrm{N}_{\mathrm{ss}}$ :, question : introduit très naturellement du discours direct. En ce qui concerne la dépendance, écueil qui est un mot peu fréquent (26 occurrences au total) est employé dans la construction avec des numéraux...nous gardant de trois écueils :; reste à éviter deux écueils :

Dans $N_{s s}$ réside/consiste en, on remarque que problème et consister sont en relation de collocation. Dans avoir pour $N_{s s,}$ l'attraction concerne principalement les aléthiques et les bouliques, plus marginalement les axiologiques. En revanche, ceux-ci sollicitent fréquemment la construction (mérite, courage, honneur...), ainsi que les deux épistémiques sentiment et certitude. Finalement, on voit que dans est un $N_{s s}$, en dépendance, les appréciatifs sont nombreux.

L'analyse entreprise ici demanderait une place plus large pour apprécier de façon exhaustive la distribution et le fonctionnement discursif non seulement des Nss mais aussi des constructions. À ce stade, l'important a été de montrer qu'une perspective essentiellement modale (les

${ }^{9}$ Cf. Lenepveu (2009), Huyghe (ici même). 
catégorisations existantes ne sont que partiellement modales) s'avère pertinente dans le travail de caractérisation de la dépendance et de l'attraction entre noms et constructions.

\section{POUR CONCLURE}

Cette étude a examiné l'usage des Nss dans une pratique langagière particulière. Comme la variété des pratiques socio-discursives et des régularités micro-linguistiques selon les types de texte et les genres discursifs (Adam, 2001) influe sur les propriétés statistiques d'un corpus, il serait instructif de contraster l'utilisation des Nss dans des types de textes et genres discursifs différents. Une entreprise pareille serait révélatrice pour - entre autres - déterminer non seulement la variation de fréquence des Nss selon les genres, mais aussi leur distribution dans les différentes constructions ainsi que la fréquence également de ces constructions. La recherche n'a pas encore atteint ce stade d'investigation et donc beaucoup reste à faire dans ce domaine. Nous espérons que cette contribution aura éclairé un pan et ajouté une modeste strate au savoir accumulé.

Dans une étape ultérieure, il serait instructif de creuser la question du rôle textuel des Nss, en examinant les relations de cooccurrence entre Nss et en dégageant les résultats au niveau global du corpus : tel Nss a-t-il tendance à être employé avec tel(s) autre(s) Nss ? Si cooccurrence il y a, celle-ci est-elle structurée par une séquence d'enchaînement ? Par exemple, si les mots problème et stratégie ont tendance à être employés ensemble, font-ils partie de la même séquence d'enchaînement? Il s'agirait donc d'examiner comment non pas un Nss (ce qui a été entrepris jusqu'à maintenant), mais des relations entre Nss participent à la construction textuelle - tout en prenant en compte le poids de chaque construction. Les cooccurrences entre Nss pourraient aussi être corrélées ultérieurement à des textes génériquement différents pour vérifier si tel genre se distingue d'un autre par l'intégration de cooccurrences typiques.

\section{Références}

ADAM J.M. (2001), « Types de textes ou genres de discours ? Comment classer les textes qui disent de et comment faire ? », Langages 141, 10-27.

ADLER S. (2012), "Trois questions relatives aux noms généraux factuels attitudinaux », Scolia 26, 11-37.

ADLER S. (2017), «Les noms généraux - 'shell nouns' - participent-ils à une lecture taxinomique de type hiérarchie-être ? », Syntaxe et Sémantique 18, 45-66.

ApOTHELOZ D. (2008), « À l'interface du système linguistique et du discours : l'exemple des constructions identificatrices », in O. Bertrand et al. (éds), Langue, discours et diachronie, Berne : Peter Lang, 75-92.

BLANCHE BENVENISTE C. (1992), «Sur un type de nom 'évaluatif' portant sur des séquences verbales », Review of applied linguistics 97-98, 1-25.

FlauX N. \& VAN DE VelDE D. (2002), Les noms français, esquisse de classement, Paris : Ophrys.

Flowerdew J. \& FoREST R. (2015), Signalling Nouns in English: A Corpus-based Discourse Approach, Cambridge University Press.

Gosselin L. (2010), Les modalités en français: la validation des représentations, Amsterdam : Rodopi.

GRIES S. (2013) "50-something years of work on collocations : What is or should be next ...", International Journal of Corpus Linguistics 18:1, 137-165.

HALlidAY M .A .K. \& HASAN R. (1976), Cohesion in English, London: Longman.

HuYGHE R. (2015), «Les typologies nominales : présentation », Langue française 185/1, 527. 
LEGALlois D. (2006), «Quand le texte signale sa structure : la fonction textuelle des noms sous-spécifiés », Corela, HS-5/ 2006, mis en ligne le 27.10.2006. http://corela.revues.org/1465

LEGALloIS D. (2008), «Sur quelques caractéristiques des noms sous-spécifiés », Scolia 23, 109-127.

Legallois D. \& GREA Ph. (2006), «L'objectif de cet article est de... Construction spécificationnelle et grammaire phraséologique », Cahiers de Praxématique 46, 161-186.

LEGALlois D. \& LENEPVEU V. (2014), «L'évaluation dans les textes : des relations interpropositionnelles aux séquences discursives », Langue française 184/4, 17-33.

LENEPVEU V. (2009) «À propos de l'expression nominale le fait est que », Le français moderne LXXVII-2, 199-218.

Lyons J. (1977), Semantics, Cambridge: Cambridge University Press.

MAHLberg M. (2005), English General Nouns, Amsterdam: John Benjamins.

NAKAMURA T. (2017), «Extension transitive de constructions spécificationnelles », Langue Française, 194, 69-83.

Roze Ch., Charnois Th., Legallois D. Ferrari S. \& Salles M. (2014), «Identification des noms sous-spécifiés, signaux de l'organisation discursive ». Actes de la $21^{\text {ème Conférence }}$ sur TALN, Marseille, 377-388.

SCHMID H.-J. (2000), English Abstract Nouns as Conceptual shells, New York: Mouton de Gruyter.

SCHNEDECKER C. (2006), «Anaphores prédicatives démonstratives: de la cohésion syntagmatique à la cohérence textuelle », Corela mis en ligne le 27.10.2006, http://corela.revues.org/1437.

Vernier M., Mathet Y., Rioult F., Charnois Th., Ferrari S. \& Legallois D. (2007), "Classification de textes d'opinions : une approche mixte n-grammes et sémantique » Actes du $3^{e}$ défi fouille de texte, Grenoble, 3 juillet 2007, 105-119.

WINTER E. O. (1992), "The notion of unspecific versus specific as one way of analyzing the information of a fund-raising letter", in W.C. Mann \& S.A. Thompson (eds), Discourse descriptions, Amsterdam: John Benjamins, 131-170. 